\title{
Numerical modeling of ion exchange waveguide for the tasks of quantum computations
}

\author{
V. Gerasimenko, N. Gerasimenko, F. Kiselev, E. Samsonov, S. Kozlov \\ ITMO University, Kronverkskiy, 49, Saint Petersburg, 197101, Russia \\ lyagacruz@gmail.com
}

DOI 10.17586/2220-8054-2019-10-2-147-153

\begin{abstract}
This paper is devoted to the simulation of a single-mode ion-exchange waveguide and the $3 \mathrm{~dB}$ directional coupler for quantum chips. We performed diffusion modeling of $\mathrm{Na}^{+} \leftrightarrow \mathrm{K}^{+}$ions in the $\mathrm{R}_{2} \mathrm{O}-\mathrm{SnO}_{2}-\mathrm{SiO}_{2}$ glass and optical modeling by the beam propagation method. A wavelength of $1064 \mathrm{~nm}$ was used corresponding to the requirements of the single-mode regime for our waveguide. Simulation of diffusion has shown that the profile of the refractive index of overlapping areas can be modeled by summing two separate profiles, which is crucial for optimizing performance. In the process of optical modeling it was possible to minimize losses on s-bends of changing the width of the bend and reducing the interaction length to zero. So we looked at many aspects of device optimization and performed a design, manufacture and characteristics simulation of a directional $3 \mathrm{~dB}$ coupler. The overall transmittance of proposed device was evaluated as 0.96 .
\end{abstract}

Keywords: directional coupler, integrated waveguide, quantum computation, integrated optics.

Received: 11 March 2018

Revised: 2 April 2019

\section{Introduction}

Integrated quantum photonics is a well-known physical platform for implementation of quantum computing. This technology uses principles of linear optics which were shown to be suitable for such tasks since a photon can exist in a superposition of two optical modes (spatial or polarization) and thus serve as a qubit. This approach is called dual-rail encoding and perfectly maps on photonic integrated circuits. Key elements of any linear optical quantum scheme are beamsplitters and phaseshifters.

Almost all modern devices of integrated quantum photonics are produced by lithography, growing on the surface of a crystal or etched semiconductor membranes [1-6]. All of these methods are extremely complicated and expensive, and in addition, all have significant drawbacks.

Etched semiconductor membranes are one of the most exotic quantum photonics materials, as crystals can be generated with the required dimensions. In addition its properties can be modified by doping [6]. At the same time, fabrication of membranes is a very complicated and expensive process, and, being non-coated, is very sensitive to environmental properties. Furthermore waveguides can be very fragile and easily damaged with the slightest manipulation.

Grown crystal waveguides are easier to manufacture and are less fragile then previous ones, but can be made with the same accuracy as membranes [5]. The main problem with these waveguides become apparent when one try to produce thermally or electromagnetically-controlled active elements. This is difficult because of the required optical insulation of these devices and waveguides.

Lithography is one of the most common processes for producing optical quantum computation devices $[1,4]$. In comparison with etched membranes and grown crystal waveguides, lithographic ones are much rougher; for this technology, it is impossible to control the device's dimensions to nanometer-level specifications. However, this method is suitable for glasses with small refractive index contrast, so characteristic size of waveguide can be increased to micrometers, for which lithography error becomes negligible. In addition there are no problems with burying these waveguides, and the optical insulation task can be solved easily. Additionally, for a waveguide with sharp refractive index profile, one can determine the mode composition at a given wavelength using relatively simple calculations. On the other hand, for every optical quantum chip, it is crucial to ensure single mode propagation in an area where active element works, so sizes of waveguide and its border defects are close, this can be a source of relatively significant light scattering and losses.

The ion exchange process makes it even easier to obtain a buried waveguide chip, and this device would have specified parameters suitable for feeding through a fiber [7]. An Ion exchanged waveguide has a gradient refractive index profile, which has fewer losses and scattering problems, and thus don't impede proper device operation. Theoretically, it is possible to obtain single-mode gradient waveguide by ion exchange for wavelengths about $1.55 \mu \mathrm{m}$. 
In this paper, we will consider the ion exchange of $\mathrm{Na}^{+} \leftrightarrow \mathrm{K}^{+}$in three-component glasses with the composition $\mathrm{R}_{2} \mathrm{O}-\mathrm{SnO}_{2}-\mathrm{SiO}_{2}$, since their optical properties shift [8] and parameters of the diffusion process [9] are known. At the same time, the sizes of the exchanged ions and the strengths of their fields are relatively similar, which allows one to ignore some effects specific for dissimilar ions [10].

This paper describes the numerical simulation of a single-mode ion-exchange waveguide and directional coupler for quantum chips.

\section{Diffusion modeling}

The first step in modeling of waveguides is the definition of its refractive index profile. This can be obtained via the modeling of the ion-exchange process. In this process, the longest stages are the diffusion of substituting ions into a glass and the displaced ones from a glass [8], so it is the first step to simulate these processes at the stage of production and burying of the waveguide.

Consider the case of ion exchange in thermally-stable glass, to which the mask is applied. In this case, the diffusion coefficient $(D)$ can be considered constant and equal to the smaller of these coefficients for the exchanged ions. The source of substituting ions for creation of a waveguide is set by the boundary conditions: the concentration of these ions $(C)$ on the non-masked part of the glass is constant and equal to the total concentration of alkaline ions in the studied glass. At the waveguide burying stage, the mask is removed, and the entire boundary is covered by the source of initial ions.

The diffusion equation takes the form:

$$
\frac{\partial C}{\partial t}=D \nabla^{2} C
$$

boundary conditions at the stage of obtaining the waveguide:

$$
\begin{array}{ll}
C=1, & \text { for non-masked area } \\
\frac{\partial C}{\partial t}=0, & \text { for masked area } \\
\frac{\partial C}{\partial t}=D C, & \text { for inner boundaries }
\end{array}
$$

and at the stage of burying:

$$
\begin{array}{ll}
C=1, & \text { for melt-glass boundary } \\
\frac{\partial C}{\partial t}=D C, & \text { for inner boundaries }
\end{array}
$$

Figure 1 describes simulated area graphically.

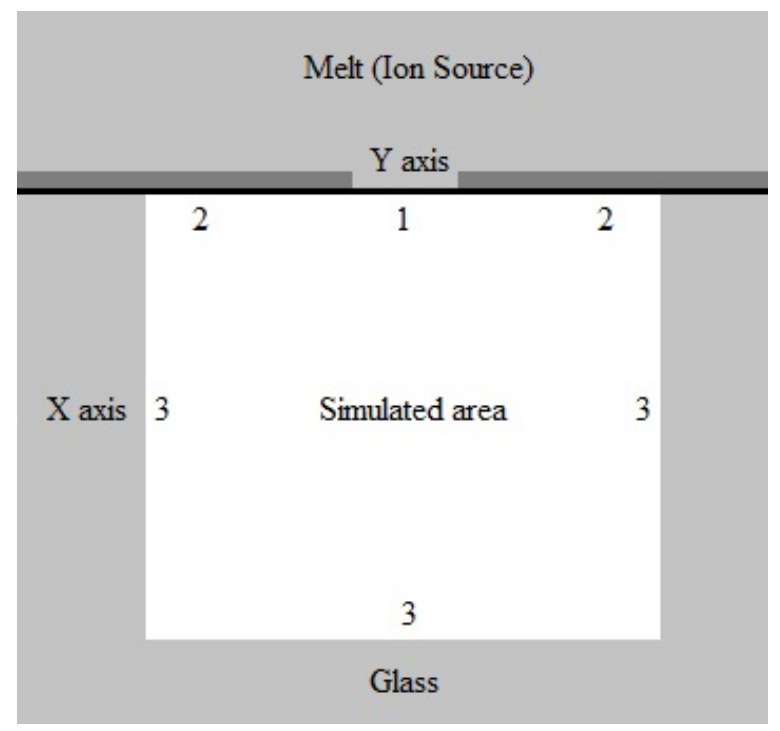

FIG. 1. Graphical description of simulated area. Space only inside white rectangle was modelled. 1 is a mark for non-masked area, 2 for masked one when waveguide is manufacturing. When it is burying both of the marks denote melt-glass boundary. 3 is a mark for inner boundaries (regardless of manufacturing stage) 
For numerical simulation, we used the explicit finite difference method on rectangle grid with spatial step of $0.1 \mu \mathrm{m}$ and time step of $0.25 \mathrm{~ns}$.

After obtaining the distribution of the concentration of substituting ions, the next stage was to determine the profile of refractive index $(n)$, which depends linearly on substituting ion concentration, as it was shown in some experimental works $[7,10]$ :

$$
n=n_{0}+\Delta n_{\max } C
$$

where $\Delta n_{\max }$ is the change in the refractive index at $C=1$ (and $\Delta n=\Delta n_{\max } C$ ), $n_{0}$ is refractive index of the glass before ion exchange (at $C=0$ ). Typical values can be found in catalogs and manuals [8].

With the ion exchange of $\mathrm{Na}^{+} \leftrightarrow \mathrm{K}^{+}$in three-component quartz glass $\mathrm{R}_{2} \mathrm{O}-\mathrm{SnO}_{2}-\mathrm{SiO}_{2}$ at a temperature of $T \approx 600{ }^{\circ} \mathrm{C}$, the diffusion coefficient is known $D \approx 3.4 \mu \mathrm{ms}$ [10]. The calculations were done for the width of the non-mask area of $2 \mu \mathrm{m}$.

Simulation of ion exchange under such conditions gives the results shown in Fig. 2.

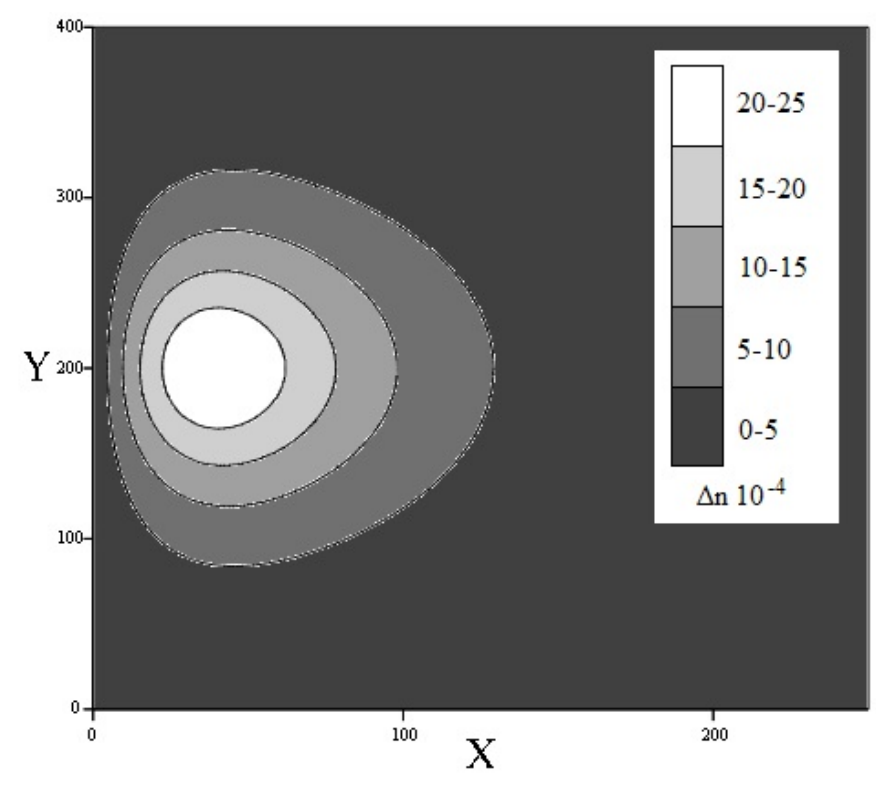

FIG. 2. The result of modeling the profile of refraction index. There was simulated a diffusion through $2 \mu \mathrm{m}$ slit in mask. The step of counts on the axes is $0.1 \mu \mathrm{m}$

The calculation of ion concentrations for the transfer region in the directional coupler was also a subject of interest. Simulation, the results of which are presented in Fig. 3 and 4, showed that it is possible to use a simple summation of concentrations when the distance between non-masked areas is $14 \mu \mathrm{m}$, because the error in determining the refractive index does not exceed $5-7 \cdot 10^{-5}$. This fact makes it possible to exclude modeling of the merger and separation areas of the waveguides in the directional coupler.

\section{Optical modeling}

Since the refractive index difference between the core of the waveguide and the cladding is quite small, we used the beam propagation method [11] to simulate our device. First, we had to calculate field and an effective refractive index of the fundamental mode of this waveguide at $1064 \mathrm{~nm}$ wavelength (Fig. 5). This wavelength corresponds to an SPDC bi-photons source [12] and also fits the requirements of a single-mode regime for our waveguide.

In this section, we will refer to various parameters of the geometry of directional coupler. In general, this geometry can be represented by the geometry of the mask used to manufacture it (Fig. 6). An important characteristic about ion-exchanged glass waveguides is that their fundamental mode aligns well with the gradient of the refractive index profile. This causes an issue in the design process for the directional coupler. Since coupling between two modes depends on the value of the overlap integral, it becomes clear that coupling will be very weak if the waveguides' gradients don't intersect. This means that in order to achieve splitting of the power between two single-mode waveguides, we need to overlap our waveguides in the interaction region. This of course comes with a drawback, as we create wide and complex refractive index profile which might cause excitation of high 


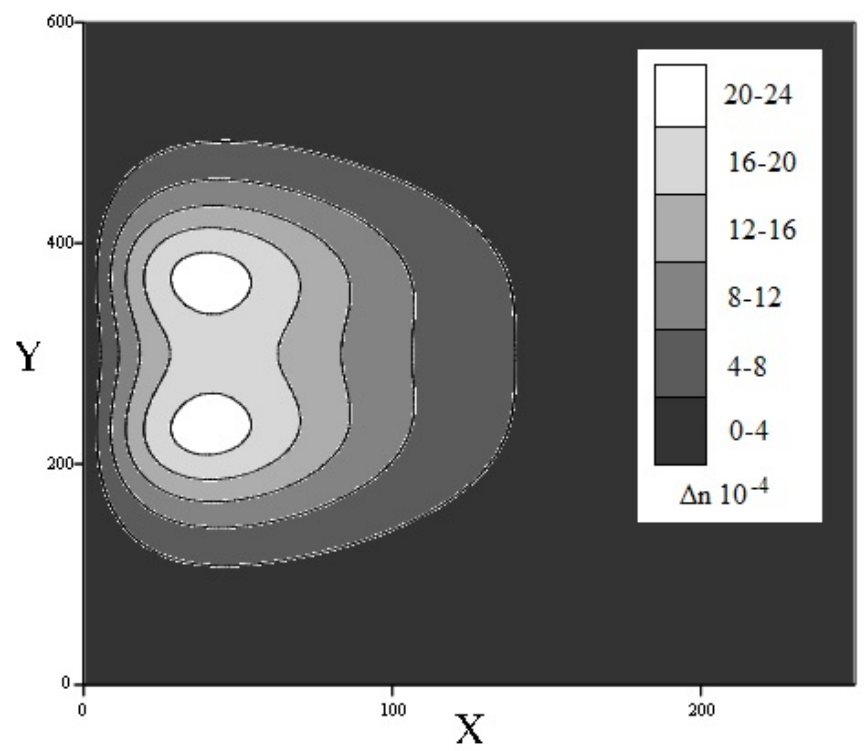

FIG. 3. The result of directional coupler modeling. Simulation was performed for two $2 \mu \mathrm{m}$ slits with $14 \mu \mathrm{m}$ of masked area between them. The step of counts on the axes is $0.1 \mu \mathrm{m}$

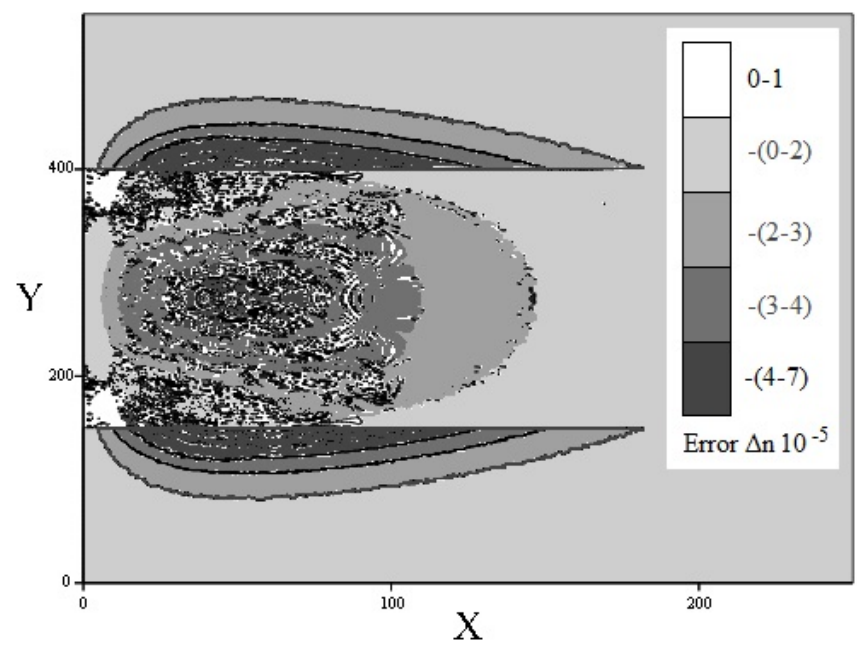

FIG. 4. A simple-summation error estimate for directional coupler. The step of counts on the axes is $0.1 \mu \mathrm{m}$. Maximum definition errors are $\Delta n=0.00007$ (at sharp boundaries, after which one component of the sum remained) and $\Delta n=0.00005$ (in the center)

order modes. Effectively, this could result in an additional loss of power (Fig. 7), as higher order modes of this region will have much less efficient coupling to single mode output waveguides.

Curvatures and leakage from the fundamental modes might occur at these bends (Fig. 8). In the optimization process, we decided to utilize the zero length for the so-called interaction region, meaning that very close overlap between the waveguide will remain only in one point. We observed that in this regime, we obtained fewer losses associated with the multi-mode regime of such overlap. In order to optimize the losses associated with s-bends, we decided to vary values for the initial separation and separation in the interaction region to determine whether we could reduce these losses while preserving overall length of the device and its splitting coefficient. Simulation of optimized design is shown on Fig. 9. One can see by the solid line on the value monitor that losses are significantly less than in cases shown on Fig. 7 and 8 . The overall transmittance of the device is $\sim 0.96$ with the length of $20 \mathrm{~mm}$. We were able to achieve this with initial separation of $160 \mathrm{~nm}$ and separation in the interaction region of $15.82 \mu \mathrm{m}$. We want to note the importance of the analysis presented in the first section of this article. Showing 


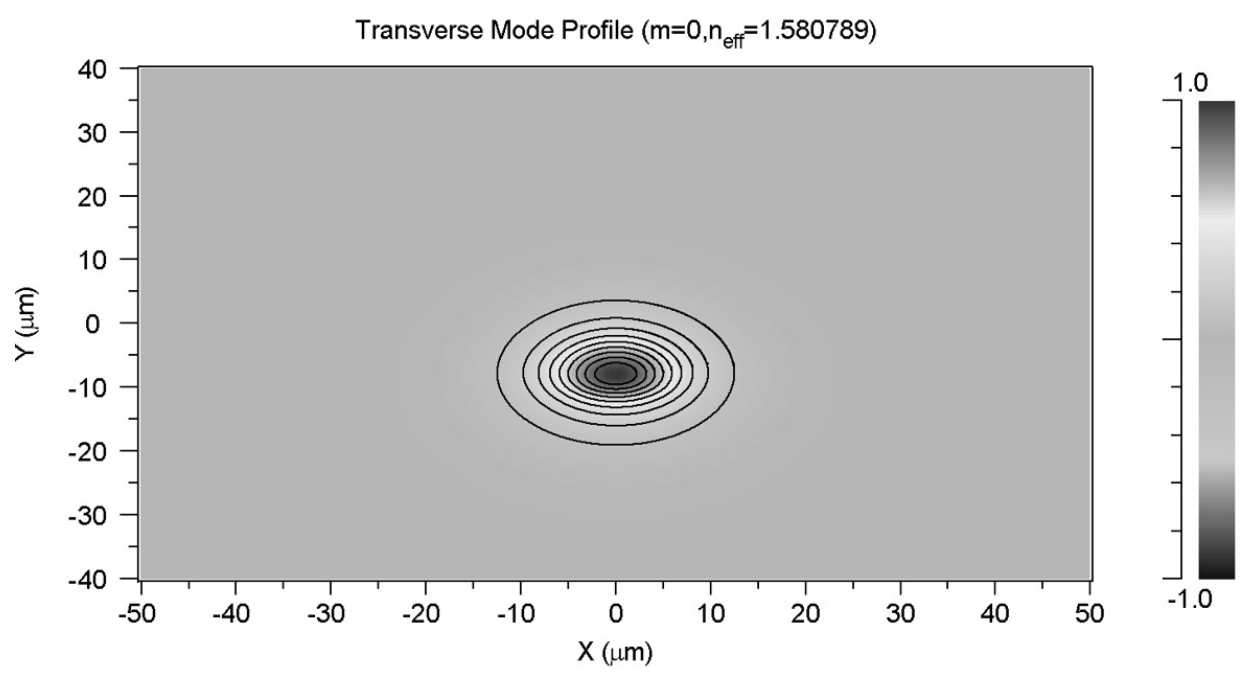

FIG. 5. Calculated fundamental mode of the ion-exchange glass waveguide with refractive index profile shown on Fig. 2

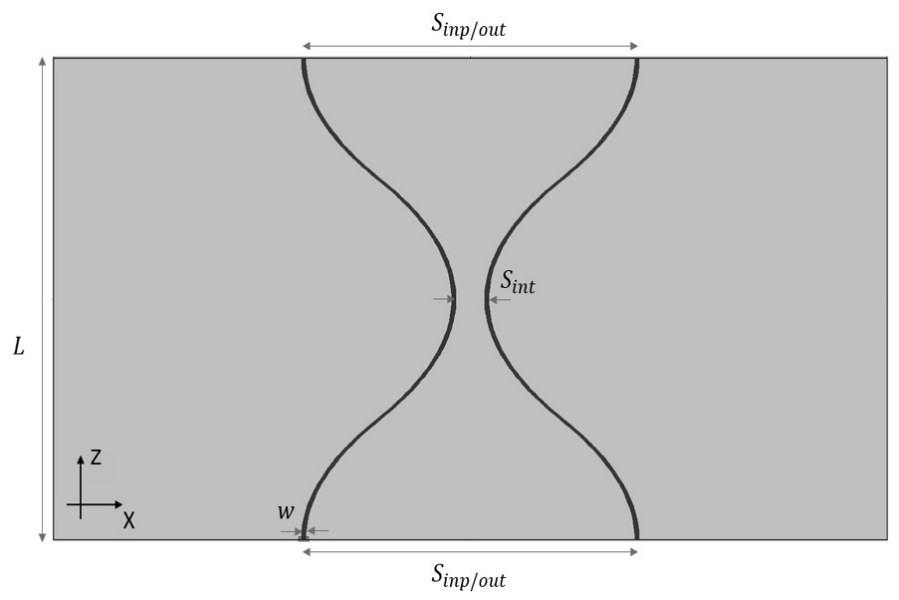

FIG. 6. Geometry of the mask for the $3 \mathrm{~dB}$ directional coupler. $\mathrm{S}_{\text {inp } / \text { out }}$ is initial and $\mathrm{S}_{\text {int }}$ is the interaction region separations, $w$ is a width of slits in the mask which is $2 \mu \mathrm{m}$. Each of the two slits correspond to left and right optical channels of the device

that we can directly sum two displaced profiles instead of performing diffusion modeling for every separation in the interaction region allowed us to significantly expedite the optimization process.

\section{Conclusion}

In this paper, we performed design, fabrication and performance modeling of the $3 \mathrm{~dB}$ directional coupler based on ion-exchanged glass waveguides. In the section dedicated to the optical modeling of the device, we showed important aspects of the design optimization. In particular, we showed how the problem of overlapping in the interaction region and its multi-mode regime can be mitigated by reducing the interaction length to zero and managing width of the s-bend. We also showed that the refractive index profile of overlapping regions can be simulated by directly summing two separated profile calculated from the modeling of diffusion process. This was extremely important in the optimization process of the device, as it saved a significant amount of time which would otherwise be spent on diffusion simulations. The proposed design of the device has length of $20 \mathrm{~mm}$ with maximum and minimum separation distances of $160 \mu \mathrm{m}$ and $15.82 \mu \mathrm{m}$ respectively. Its overall transmittance was evaluated as 0.96 which is different from ideal 1 due to light leakage from the s-bend. 

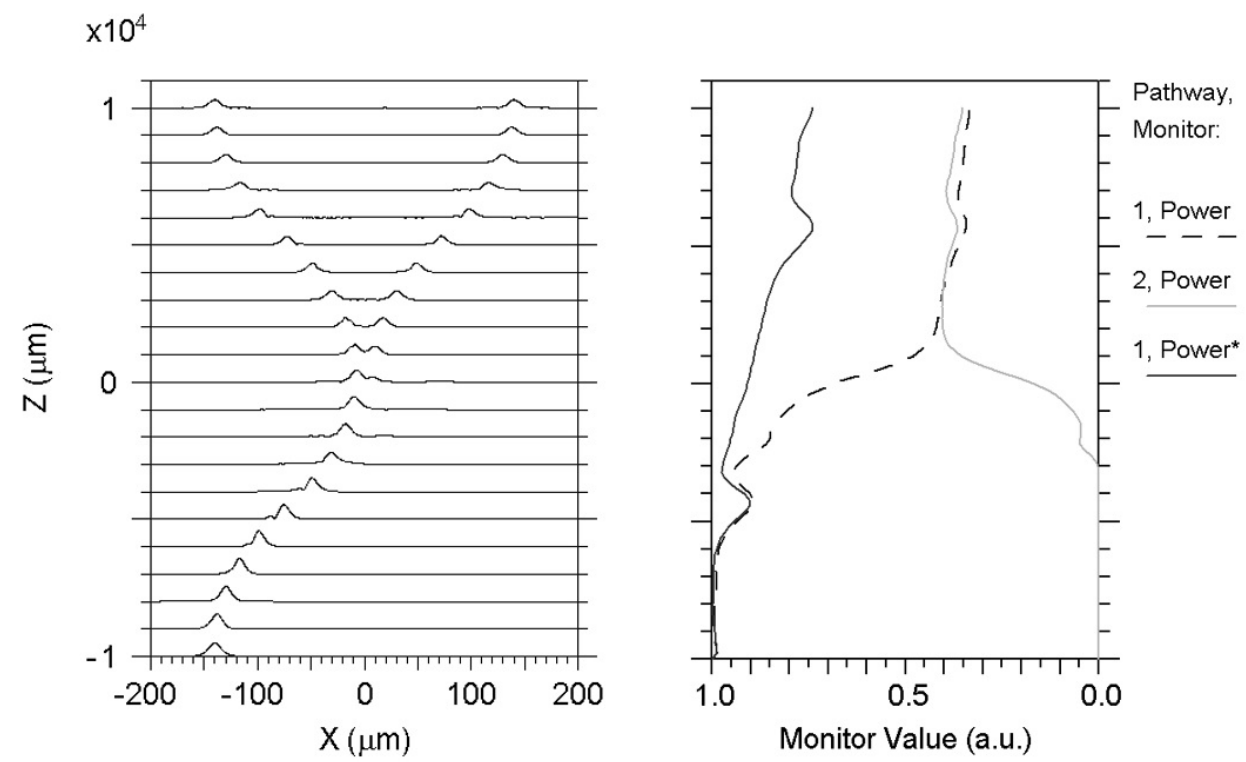

FIG. 7. Simulation of light propagation in the $3 \mathrm{~dB}$ directional coupler with overlapped interaction region. The initial separation is $280 \mu \mathrm{m}$, separation in the interaction region is $16.5 \mu \mathrm{m}$, interaction length is $500 \mu \mathrm{m}$. Left is the field contour map along the device and right picture shows power in the left channel (dashed black), power in the right channel (solid grey) and overall power in the device (solid black)
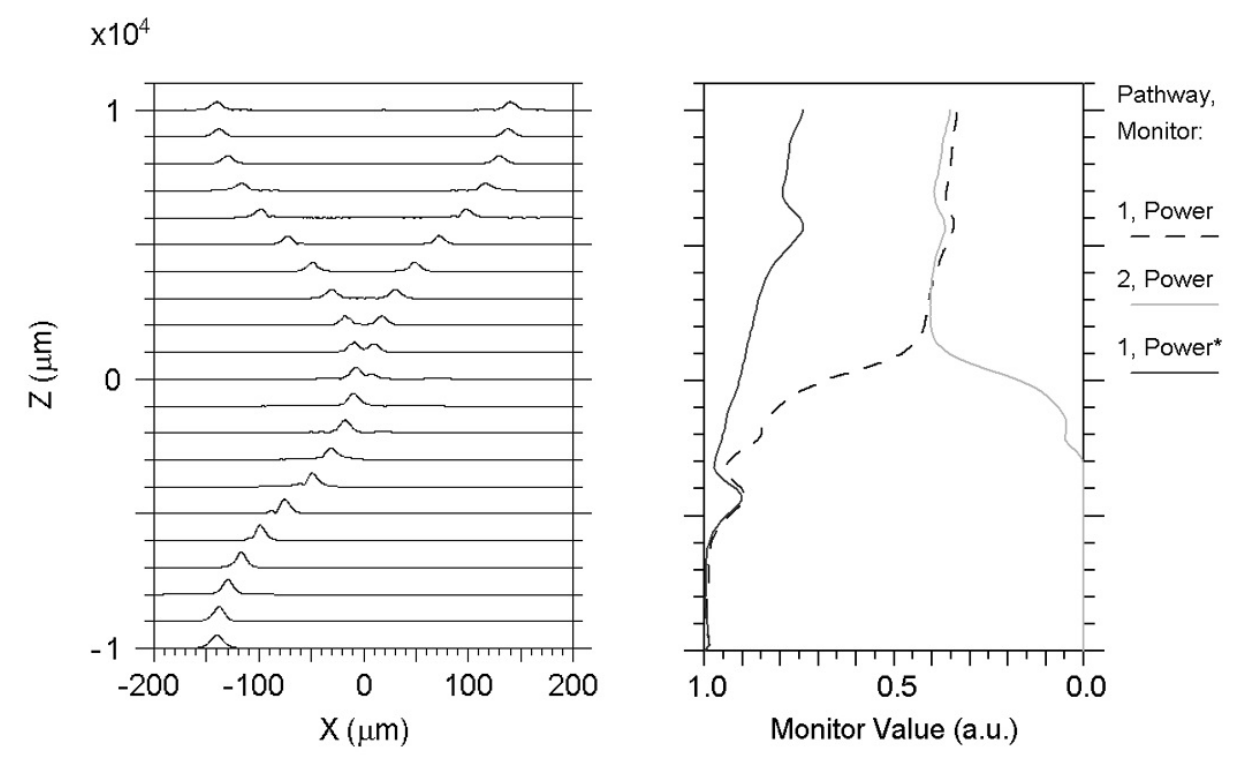

FIG. 8. Simulation $3 \mathrm{~dB}$ directional coupler with coupling strength controlled by the curvatures of s-bends and separation of their inputs. Initial separation is $280 \mu \mathrm{m}$, separation in the interaction region is $14.5 \mu \mathrm{m}$, interaction length is $500 \mu \mathrm{m}$. The left is the field contour map along the device and right picture shows power in the left channel (dashed black), power in the right channel (solid grey) and overall power in the device (solid black) 

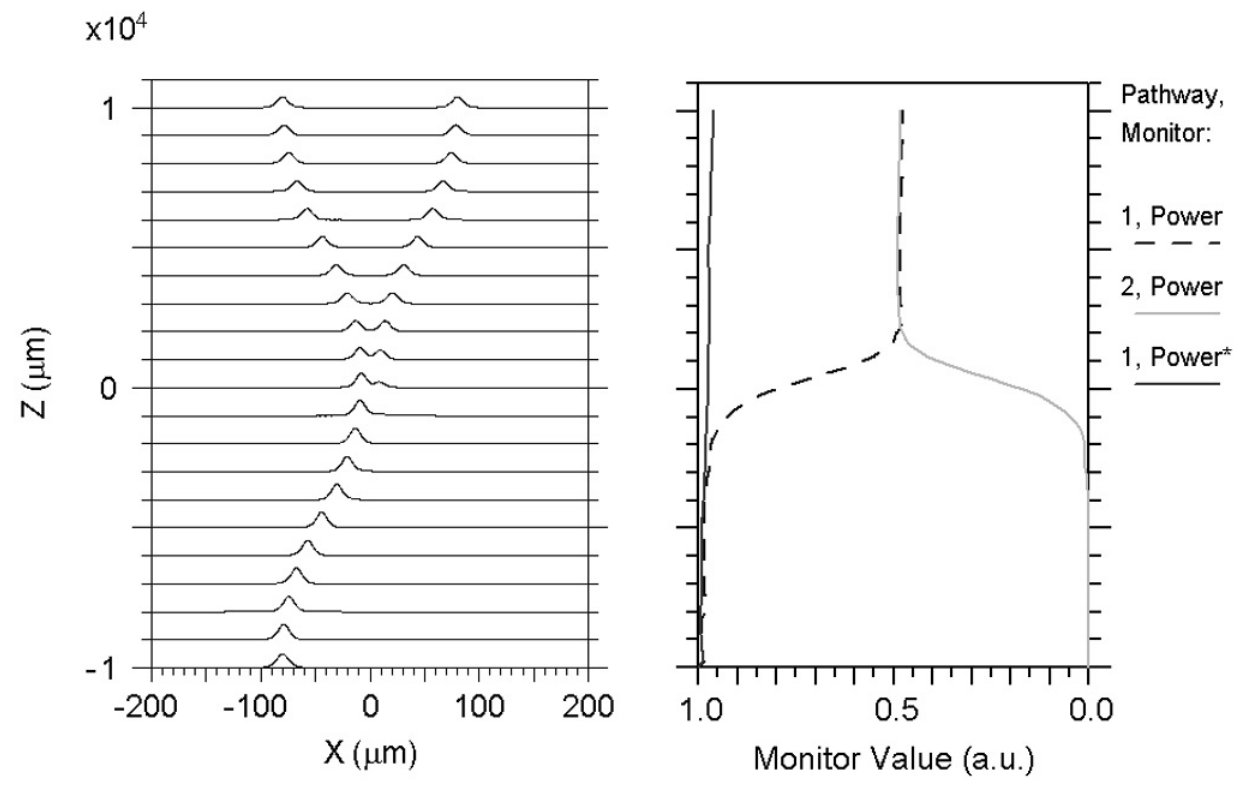

FIG. 9. Simulation of light propagation in of the optimized $3 \mathrm{~dB}$ directional coupler. Initial separation is $160 \mu \mathrm{m}$, separation in the interaction region is $15.82 \mu \mathrm{m}$ interaction length is $0 \mu \mathrm{m}$. Left is the field contour map along the device and right picture shows power in the left channel (dashed black), power in the right channel (solid grey) and overall power in the device (solid black)

\section{References}

[1] Matthews J.C.F., Politi A., Stefanov A., O’Brien J.L. Manipulation of multiphoton entanglement in waveguide quantum circuits. Nature Photonics, 2009, 3(6), P. 346-350.

[2] Crespi A., Ramponi R., Osellame R., Sansoni L., Bongioanni I., Sciarrino F., Vallone G., Mataloni P. Integrated photonic quantum gates for polarization qubits. Nature communications, 2011, 2, P. 566.

[3] Politi A., Matthews J.C.F., Thompson M.G., O’Brien J.L. Integrated quantum photonics. IEEE Journal of Selected Topics in Quantum Electronics, 2009, 15(6), P. 1673-1684.

[4] Politi A., Cryan M.J., Rarity J.G., Yu S., O’Brien J.L. Silica-on-silicon waveguide quantum circuits. Science, 2008 , 320(5876), P. 646-649.

[5] Zhang Y., McKnight L., Engin E., Watson I.M., Cryan M.J., Gu E., Thompson M.G., Calvez S., O’Brien J.L., Dawson M.D. GaN directional couplers for integrated quantum photonics. Applied Physics Letters, 2011, 99(16), P. 161119.

[6] Hallett D., Foster A.P., Hurst D.L., Royall B., Kok P., Clarke E., Itskevich I.E., Fox A.M., Skolnick M.S., Wilson L.R. Electrical control of nonlinear quantum optics in a nano-photonic waveguide. Optica, 2018, 5(5), P. 644-650.

[7] West B.R., Madasamy P., Peyghambarian N., Honkanen S. Modeling of ion-exchanged glass waveguide structures. Journal of NonCrystalline Solids, 2004, 347(1-3), P. 18-26.

[8] Nikonorov N.V., Aseev V.A., Zhukov S.N., Ignatiev A.I., Kiselev S.S., Rokhmin A.S. WAVEGUIDE PHOTONICS. SPSU ITMO, St. Petersburg, 2008, $82 \mathrm{p}$.

[9] Zhabrev V.A. Diffusion processes in a glassy coating layer. Obtaining and applying protective coatings: proceedings of the 12-th All-Union Conference on Heat-Resistant Coatings, Leningrad April 16-18, 1985, Leningrad: Science, 1987, P. 14-18.

[10] Zhurikhina V.V., Petrov M.I., Sokolov K.S. and Shustova O.V. Ion-exchange characteristics of sodium-calcium-silicate glass: calculation from mode spectra. Technical Physics, 2010, 55(10), P. 1447-1452.

[11] Yevick D., Hermansson B. Efficient beam propagation techniques. J. Quantum Electron., 1990, 26(1), P. 109-112.

[12] Magnitsky S., Frolovtsev D., Firsov V., Gostve P., Protsenko I., Saygin M. A SPDC-based source of entangled photons and its characterization. Journal of Russian Laser Research, 2015, 36(6), P. 618-629 